\title{
POSSIBILITY OF USING A RING ACCELERATOR FOR IONIZATION COOLING OF MUONS*
}

\author{
V.I.Balbekov, FNAL, Batavia, IL
}

\section{Abstract}

A possibility of using a racetrack-like ring accelerator as the first stage of a cooler for muon collider is considered. The ring cooler includes bending magnets with field index 0.5 , bent solenoids, and straight sections with accelerating cavities and solenoids. LiH rods placed in straight sections are used for transverse cooling whereas wedge absorbers in bending parts provide transverse-longitudinal emittance exchange. Results of analytical calculations and Monte Carlo simulations with scattering and straggling are presented, and problem of suppression of nonlinear and chromatic effects is discussed.

\section{INTRODUCTION}

This report examines the possibility of using a racetracklike ring accelerator in the cooling stage of muon collider (Fig.1). The main merit of such a cooler is lower projected cost because the same accelerating and focusing system is used repeatedly (about 20 turns in a typical scenario) [1].

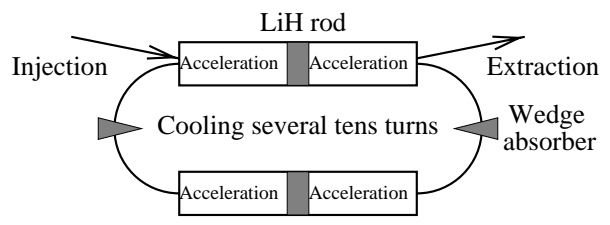

Figure 1: Schematic of a ring cooler.

'Usual' ring accelerator could provide the cooling which is schematically shown on Fig.2, left. Boundary of a beam on phase plane $\left(X-X^{\prime}\right)$ is a phase trajectory depending on $\beta$ - function and conserving the shape if compression by a cooling exist. But $X^{\prime}$ size is restricted by multiple Coulomb scattering typically $300 \mathrm{mrad}$ on $3 \sigma$ - level. Initial size cannot be significantly more because of aperture restriction, so a deep cooling is impossible in such a scheme. To provide it, it is necessary to get independent motion in $X-$ and $X^{\prime}$ - directions on phase plane as shown on Fig.1, right. It means that transfer matrix is $\pm I$; another words, betatron frequency of the ring cooler must be integer or half-integer.

It refers to synchrotron motion, too, but it is very difficult to get synchrotron frequency 0.5 at low RF. But the considered version of a ring cooler is focused on initial stage of cooling when bunch length is several meters [2, 3], and using of high RF is impossible. Hence such a cooler must operate at transition energy which formally corresponds to synchrotron frequency 0 . Bunchers are needed only for longitudinal compression of the bunch.

\footnotetext{
* Work supported by the U.S. Department of Energy under contract No DE-AC02-76CH0300
}

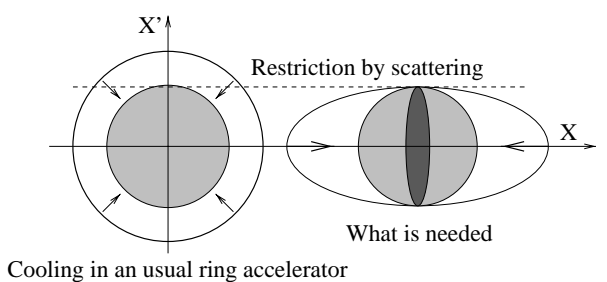

Figure 2: The cooling dynamics in 'usual' and 'resonant' ring cooler.

\section{SCHEMATIC AND PARAMETERS OF THE COOLER}

Schematic of the cooler is shown on Fig.3. LiH rods $0.31 \mathrm{~m}$ long are placed inside solenoids $2.32 \mathrm{~m}$ long. Each solenoid is divided on 2 non equal parts with opposite direction of field: (1) $L=1.142 \mathrm{~m}, B=3.207 \mathrm{~T}$, (2) $L=1.178 \mathrm{~m}$, $B=-2.646 \mathrm{~T}$, and each part provides betatron phase advance $\pi / 2$. The main destination of these solenoids is to focus very divergent beam going out the rods to parts of straight section with cavities. Four $0.5 \mathrm{~m}$ solenoids are used to focus the beam in these parts. Each solenoid with adjacent drift spaces provides phase advance $\pi / 2$. Field of the solenoid depends on its position and lies in the range 1.95 $-2.36 \mathrm{~T}$.

Bent solenoids of radius $0.75 \mathrm{~m}$ are placed inside combine function magnets with field index 0.5 . This complex provides the same focusing as central solenoid and recreates the beam size and angle divergence. Front and back bent solenoids have opposite and slightly different fields 2.105 $\mathrm{T}$ and -2.035 $\mathrm{T}$ because energy loss in center of wedge absorber is $10.7 \mathrm{MeV}$. Dipole fields are 1.489 and 1.439 T. Such a combination is very suitable for emittance exchange section because has simple transfer matrix -I and easy controlled and quite sufficient for emittance exchange dispersion function (Fig.4). It gives rather small nonlinear and chromatic distortions. A bending system without solenoid

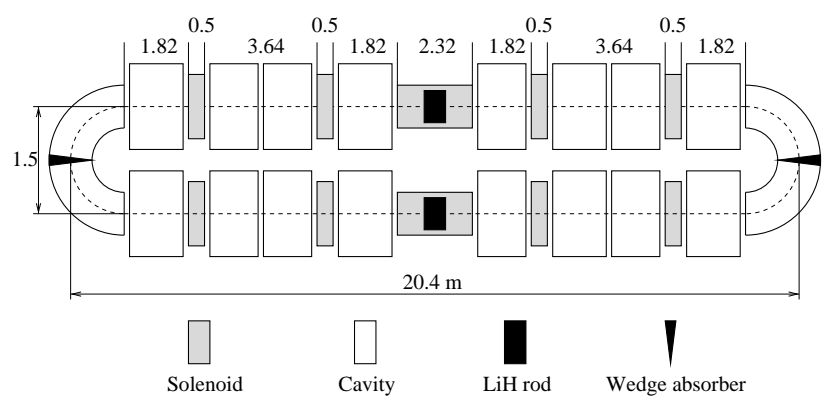

Figure 3: Schematic of a ring cooler with solenoids. 
was considered in previous version of a ring cooler [1]. In that case beam size in the bending part was essentially more resulting strong nonlinear distortions. Drawback of new system is less dispersion function resulting decrease of momentum compaction factor and growth of transition energy which is operating energy of the ring cooler. Other parameters of the cooler are listed in Table 1.

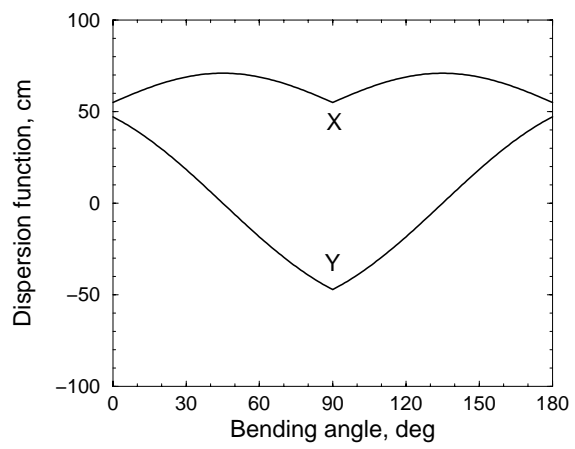

Figure 4: Dispersion function in bending part of the ring.

Table 1: Main parameters of the cooler

\begin{tabular}{|l|c|}
\hline Muon momentum, MeV/c & $297-360$ \\
Circumference, $\mathrm{m}$ & 42.47 \\
Revolution frequency & $6.167 \mathrm{MHz}$ \\
Energy gain, MeV/turn & 120 \\
Muon decay, \%/turn & 2 \\
\hline
\end{tabular}

\section{LINEAR SIMULATION}

Results of simulation in linear approximation without chromaticity are presented in this section to estimate ultimate capabilities of the cooler. Gaussian distribution with parameters: $\sigma_{x}=\sigma_{y}=7.5 \mathrm{~cm}, \sigma_{p_{x}}=\sigma_{p_{y}}=23 \mathrm{MeV} / \mathrm{c}$, $\sigma_{z}=150 \mathrm{~cm}, \sigma_{E}=11 \mathrm{MeV}$ was taken for consideration. The parameters are chosen to get constant angle and energy spread during the cooling what is really obtained.

Dependence of normalized r.m.s. emittance and transmission on number of periods is shown on Fig.5 (1 turn = 2 periods). 6-dimensional emittance decreases 2400 times by 30 turns and do not reach equilibrium yet. Transmission is is about $55 \%$ in agreement with muon decay.

Fig.6-7 give distributions of injected (left) and cooled beams on transverse and longitudinal phase plane.

\section{TRANSVERSE NONLINEARITY AND CHROMATICITY}

The beam envelopes between the rod and the wedge absorber are shown schematically on Fig. 8 before and after the cooling. In both cases there is strong modulation responsible for chromatic distortions accompanied by parametric

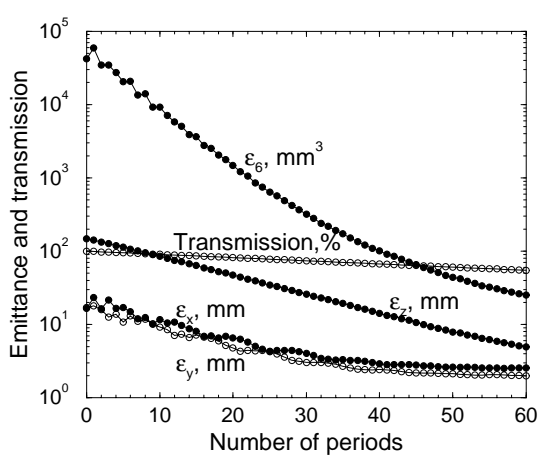

Figure 5: Dependence of emittance and transmission on number of periods.

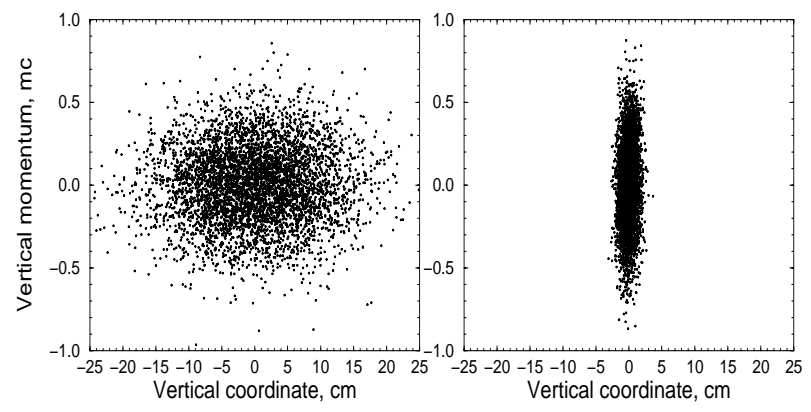

Figure 6: Initial and final transverse distributions.

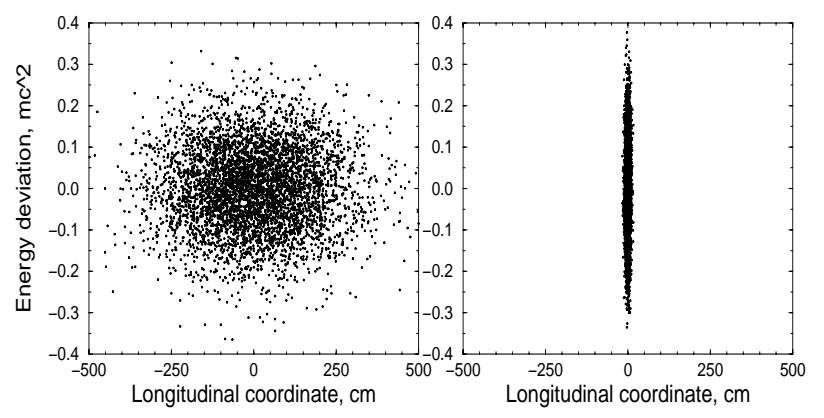

Figure 7: Initial and final transverse distributions.

resonance. The latest changes amplitude of betatron oscillations depending on them phases. Parameters of the cooler are chosen to decrease the amplitude of non-equilibrium particles going out the rod with large angle and relatively small transverse coordinate what is typical for the end of the cooling. In this case, chromatic distortions are demonstrated by Fig.9. Chromaticity is rather large still and nonlinear dispersion is observed, too. It causes transverse emittance blow up by factor 1.6 - 1.7 (see Table 2 in next section, too).

Fig.9 demonstrates transverse nonlinear effects by 1 period for equilibrium particles. Initial phase ellipses are canonical corresponding to transverse emittance on the levels $1,2,3 \sigma$. These distortions are tolerable, but it is necessary to remember that effect substantially enhanced for 
non-equilibrium particles. They give an additional emittance blow up on $10-15 \%$.

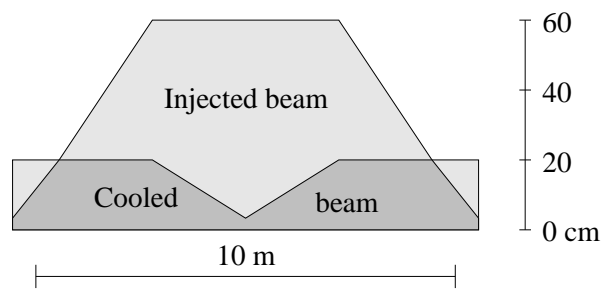

Figure 8: Beam envelop between the rod and the wedge absorber (1/4 of a turn, schematically).

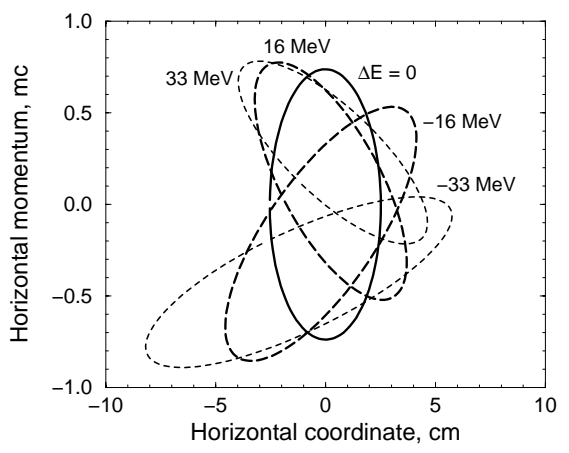

Figure 9: Phase ellipse after 1 period at different energy deviation. Initial ellipse is canonical, $\sigma_{E}=11 \mathrm{Mev}$.

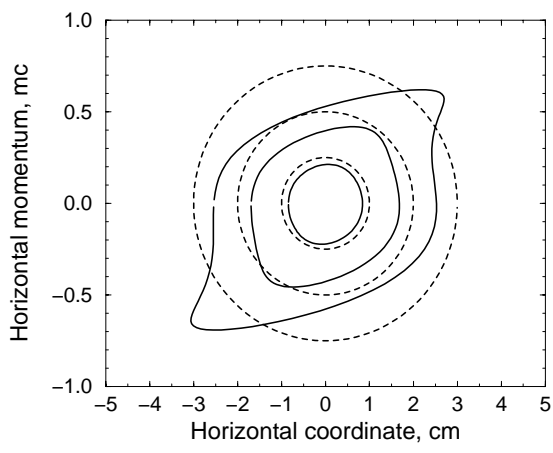

Figure 10: Nonlinear distortions by 1 period. Dashed lines - phase ellipses in the beginning (1,2,3 $\sigma$ - levels), solid lines - in the end.

\section{LONGITUDINAL NONLINEARITY}

All demonstrated results are obtained with accelerating field of harmonic number $h=1$, and almost the same turns out at $h=2$. But dependence of a trajectory length on amplitude of betatron oscillations was not taken into account yet. With it, there is significant blow up of longitudinal emittance and additional particles loss which really stop the cooling about after 16 turns. Final distributions are shown on Fig.11. It is seen that time-amplitude dependence shifts all the distribution right (average flying time increases), and

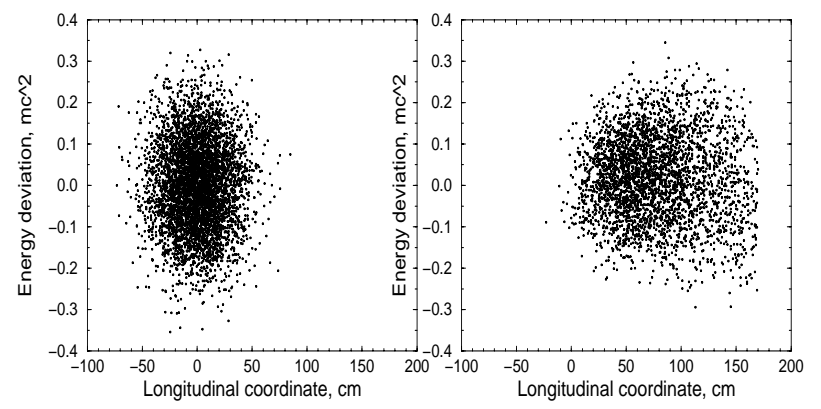

Figure 11: Longitudinal phase plane after 16 turns: linear (left) and nonlinear approximations.

a tail appears which is partially cut on the plot. After 16 turns the core of the bunch continues to compress but r.m.s. size does not change because the tail grows and the distribution becomes non Gaussian. Resulting emittances and transmission are presented in Table 2.

Table 2: Effect of different factors on the cooling (16 turns)

\begin{tabular}{|l|c|c|c|c|}
\hline & $\varepsilon_{t r}, \mathbf{c m}$ & $\varepsilon_{z}, \mathbf{c m}$ & $\varepsilon_{6}, \mathbf{c m}^{3}$ & Trans. \\
\hline Injected beam & 1.76 & 14.7 & 45.7 & 1.00 \\
\hline Linear apx. & 0.34 & 2.2 & 0.26 & 0.73 \\
+chromaticity & 0.50 & 2.4 & 0.60 & 0.62 \\
+trans. nonlin. & 0.61 & 2.7 & 1.00 & 0.57 \\
+long. nonlin. & 0.55 & 3.8 & 1.15 & 0.50 \\
\hline
\end{tabular}

\section{CONCLUSION}

The low-frequency ring cooler appears capable of satisfactory cooling a muon beam both in transverse and longitudinal directions. The achievable emittance suggests its use as a precooler in a muon collider complex especially for effective bunch shortening which is necessary in any scenario. The most serious problems are:

- Chromatic effects are very strong yet. Probably, a combined system with solenoids, quads and sextupole correctors should be developed to reduce it.

- Time-amplitude correlations are the most limiting factor for the longitudinal cooling. It is possible, special form of absorbers can help to suppress it.

- Development of low-frequency high gradient accelerating system is necessary in any case.

\section{REFERENCES}

[1] V.I. Balbekov and A. Van Ginneken, Ring Cooler for Muon Collider. AIP Conf. Proc. 441, 310 (1997)

[2] R. Palmer, Progress on $\mu^{+} \mu^{-}$Collider. Proc. PAC 97 (Vancouver, Canada, 1997), p.286.

[3] D. Neuffer and A. Van Ginneken, Muon Collection Channel. Fermilab Pub-98/296 (1998). 